\title{
Experience with Thinning: What Factors Contribute to Contradicting Results?
}

\author{
Richard P. Marini \\ Department of Horticulture, Virginia Polytechnic Institute \& State University, Blacksburg, VA 24061
}

Results from chemical thinning in commercial orchards and in experimental plantings often vary. Results from the same treatments may vary from year to year and from one location to another within a year. There are many factors that may be responsible for the differing results, but we really do not understand these factors or their potential interactions. We likely have not yet identified all the factors that affect chemical thinning. Other participants will address many of the factors affecting thinning. In this paper I will discuss some other factors that are rarely considered, but may explain many of the conflicting reports in the scientific literature. Researchers record different types of data and perform different types of statistical analyses. I will show that this may, in part, be responsible for conflicting results or differing interpretations of results that are actually similar.

\section{POLLINATION}

There are two species of parasitic mites that essentially have eliminated wild honeybees in the mid-Atlantic region and have drastically reduced the number of hives available from beekeepers in Virginia. This raised some concerns about apple (Malus $\times$ domestica Borkh.) fruit set and the activity of our chemical thinners on fruits with poor pollination.

In 1996, we performed an experiment to try to determine if thinners were more effective on fruits with few seeds. During the pink stage, varying numbers of stigmas were removed from king flowers on 'York Imperial' trees. Some trees were sprayed with carbaryl when mean fruit diameter was $\approx 10 \mathrm{~mm}$, and another set of trees were not sprayed with a chemical thinner to serve as a control. Fifty days after treatment, the number of treated fruits that remained on the tree was recorded and expressed as a percentage of the original number of treated flowers. Fig. 1 shows that fruit set was $30 \%$ for the control flowers with no stigmas removed and flowers with three stigmas removed set $22 \%$. Removal of four stigmas reduced fruit set to $12 \%$. Trees that were thinned with carbaryl had $8 \%$ to $14 \%$ fruit set regardless of the number of stigmas removed. Based on this preliminary experiment, it seems that poorly pollinated flowers set fewer fruits when not thinned, but the severity of drop following a thinning treatment may not be related to the degree of pollination. When pollination is considered inadequate, one may want to delay the application of thinners as long as possible to evaluate natural fruit drop. We have found that carbamates will provide fairly good thinning when mean fruit diameter is $\leq 14 \mathrm{~mm}$ and ethephon will provide thinning when fruit diameter is $<30 \mathrm{~mm}$.

\section{DATA COLLECTION AND ANALYSES}

Temperature and fruit size. Pomologists do not agree on the relative importance of fruit size at the time of treatment and of ambient temperature on the day of and for several days following treatment. Although there are some reports on the effects each factor individually, I am not aware of any reports where the two factors were considered together. During the past 12 years, I have performed a number of thinning experiments where mean fruit diameter and temperature were recorded. Although the experiments often differed (treatments may have been applied to whole trees or single limbs, and treatments may have been applied with a handgun or an air-blast sprayer), carbaryl was always included as a standard treatment. Multiple regression was used to evaluate the individual and interactive effects of fruit size and temperature on thinning results. The response variable was " percent fruit set" of a carbaryl treatment relative to the nontreated control based on blossom and fruit counts on

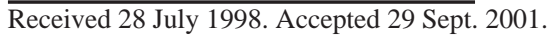

two or three limbs per tree. Percent fruit set was considered $100 \%$ if the number of fruit per 100 blossom clusters was the same for carbaryl-treated trees as for the control trees; fruit set was $50 \%$ if the carbaryl-treated trees had half as many fruit per 100 blossom clusters as nontreated control trees. The regressor variables included linear and quadratic terms for fruit diameter, the maximum, minimum, and mean temperature the day of treatment, and 1 or $2 \mathrm{~d}$ before and after treatment, the mean temperature for $2 \mathrm{~d}$ before or after treatments, the mean temperature for the 5 -day period of the $2 \mathrm{~d}$ before plus the day of plus the $2 \mathrm{~d}$ after treatment, as well as the temperature at the time of treatment. Additionally, all possible two-way interaction terms were developed for the above regressor variables.

There are a number of ways to analyze this type of a data set with regression. Sometimes, simple linear or polynomial regression is used to test each regressor variable separately. Sometimes, multiple linear regression is used to test several regressor variables, and sometimes, interaction terms are included in the models. Sequential-variableselection procedures, such as foreword selection, stepwise regression, or backward elimination, often are used to select multiple regression models. More recently, with the development of high-speed computers with adequate memory, the "all-possible-regressions method" is gaining favor among statisticians. This method generates output for all possible one-variable models, all possible two-variable models, all possible three-variable models, and so on. The researcher can request statistics such as the $R^{2}$, adjusted $R^{2}$, MSE, and Mallow's Cp to select several candidate models for further evaluation. After testing the assumptions for homogeneous residuals, normality of residuals, and non-colinearity, a model that makes biological sense can be selected. This approach is quite time consuming and it is somewhat arbitrary, but it most often allows the researcher to choose the "best" model. Table 1 shows the models selected using simple linear regression, and multiple linear regression with interaction using the Foreword option and the maximum- $R^{2}$ option of SAS's REG procedure. The final model presented was selected using the all-possible regressions method.

The multiple regression models in Table 1 show that very different models are selected depending on the method used to develop the models. The final model developed with the "all possible regressions" technique has the highest $R^{2}$ value, the highest adjusted $R^{2}$ value, the smallest sum of squared residuals, and the smallest $P$-value, and plots of partial residuals for each regressor variable do not indicate that additional variables should be included in the model. Therefore, this likely is the best model. The best model includes the mean temperature

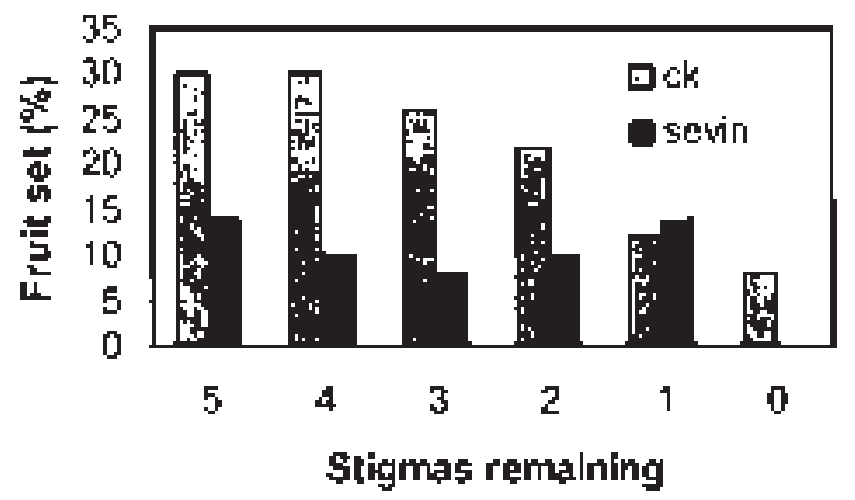

Fig. 1. Influence of carbaryl for thinning 'York Imperial' apples when varying numbers of stigmas were removed at the pink stage of bloom development. Fruit set is the percentage of treated flowers that set fruit. 
Table 1. Regression models for predicting crop load, expressed as a percentage of a nontreated control. Regressor variables included the mean fruit diameter at treatment time, maximum, minimum, and mean temperatures on the day of treatment and for one and two days before and after treatment. Several methods were used to generate regression models with SAS. ${ }^{2}$

\begin{tabular}{|c|c|c|}
\hline Predictive model & $R^{2}$ & $P$-value \\
\hline $\begin{array}{l}\text { Maximum } R^{2} \text { procedure with only linear terms } \\
\quad \text { Fruit set }(\%)=35.9+1.05 \max _{0}+1.12 \text { diam }-1.37 \min _{0}\end{array}$ & 0.31 & 0.048 \\
\hline $\begin{array}{l}\text { Maximum } R^{2} \text { procedure with linear terms and interactions } \\
\quad \text { Fruit set }(\%)=119.6-1.5 \min _{0}+0.12\left(\text { diam }^{*} \min _{+1}\right)-0.1\left(\text { diam }^{*} \min _{+2}\right)\end{array}$ & 0.35 & 0.028 \\
\hline $\begin{array}{l}\text { Maximum } R^{2} \text { procedure with only linear and squared terms } \\
\text { Fruit set }(\%)=135-4.8 \text { diam }+0.22 \text { diam }^{2}-0.78 \max _{+2}\end{array}$ & 0.43 & 0.007 \\
\hline $\begin{array}{l}\text { Maximum } R^{2} \text { procedure with linear and squared terms and interactions } \\
\text { Fruit set }(\%)=344-6.4 \max _{+2}+0.4 \max _{+2}^{2}-0.09\left(\text { diam }^{*} \min _{-2)}+0.003\left(\operatorname{diam}^{2 *} \max _{+1}\right)-0.0004\left(\operatorname{mean}_{0} * \operatorname{mean}_{+1}\right)\right.\end{array}$ & 0.61 & 0.002 \\
\hline $\begin{array}{l}\text { Foreword stepwise procedure with linear and squared terms and interactions } \\
\qquad \begin{aligned} \text { Fruit set }(\%)= & 221.8-3.2 \operatorname{mean}_{0}-0.6 \max _{+2}-0.01 \operatorname{mean}_{+1}+0.02 \max _{0}^{2}-0.005 \max _{+1}^{2}-0.09\left(\operatorname{diam}^{*} \min _{-2}\right) \\
+ & 0.003\left(\operatorname{diam}^{2 *} \max _{+1}\right)\end{aligned}\end{array}$ & 0.76 & 0.0003 \\
\hline $\begin{array}{l}\text { All possible regressions with linear and squared terms and interactions } \\
\qquad \begin{aligned} \text { Fruit set }(\%)= & 33.3+4.5 \operatorname{mean}_{0}+0.01 \max _{+1}-0.007 \max _{+1}^{2}-4.46 \min _{0}-1.4 \operatorname{diam}_{-1} 0.42 \operatorname{diam}^{2} 0.006\left(\operatorname{diam}^{2 *} \max _{+2}\right) \\
& +0.0004\left(\operatorname{diam}^{2 *} \max _{+2}^{2}\right)+0.126\left(\operatorname{diam}^{2 *} \min _{+1}\right)-0.001\left(\operatorname{diam}^{2 *} \min _{+1}{ }^{2}\right)\end{aligned}\end{array}$ & 0.85 & 0.0003 \\
\hline
\end{tabular}

positive subscripts indicate days after treatment.

on the day of treatment, the maximum temperature the day after treatment, the maximum temperature $2 \mathrm{~d}$ after treatment, the minimum temperature the day after treatment, and fruit diameter, and some of the temperature variables interact with fruit diameter. Because this model explains $85 \%$ of the variation in fruit set relative to the nontreated control, the interaction of these factors should be an area of investigation in the future.

The interaction between mean fruit diameter and temperature for the $2 \mathrm{~d}$ after treatment would be an obvious reason to expect thinning results to vary from year to year, and different results should even be expected within a year if thinning treatments are applied on different dates in the same orchard.

Factorial experiments with sub-sampling. Researchers often perform similar experiments, but record different types of data or analyze the data differently. Although usually overlooked, these may be important reasons that different researchers may obtain different results from similar experiments. Below I will demonstrate how one may arrive at different conclusions simply by estimating treatment responses differently or by using different statistical analyses.

Harvesting entire trees to determine yield, fruit size, and indices of fruit quality is expensive, and many pomologists use various types of fruit sampling methods to estimate the response variables of interest. In 1997, we used two sampling methods to estimate mean fruit weight (FW) and compared them with the true FW obtained by harvesting the entire crop. The thinning experiment was a factorial arrangement of four concentrations of methyl N', N'-demethyl-N [(methylcarbomoyl) oxy]-1-thiooxamimidate (oxamyl) and four concentrations of 1naphthaleneacetic acid (NAA). Each of the 16 treatments was applied to three trees in a completely randomized design to give a total of 48 trees. Three trees were not harvested because crows damaged a high percentage of the crop. Additionally one limb on each of three trees was lost to breakage. Therefore, as often is the case, the experiment became unbalanced. The trees were 10-yr-old 'Mercier Redchief'/M.26 trained as central leaders.

The first method (20-fruit sample) used to estimate FW involved walking around each tree and harvesting 20 fruit. Fruits were carefully selected in an attempt to represent fruits typical of those on that tree. Fruits were selected from $\approx 1$ to $2 \mathrm{~m}$ above ground and from within one meter of the tree periphery. To estimate FW for the tree, the weight of the 20-fruit sample was divided by 20 . The second method (limb samples) used to estimate FW involved harvesting all fruits on each of three limbs per tree. These limbs were $\approx 2 \mathrm{~cm}$ in diameter and were flagged during bloom to determine the number of fruits that set per 100 blossom clusters. These limbs originated $\approx 1.5 \mathrm{~m}$ above ground and typically carried $\approx 30$ to 70 fruit. At harvest, mean FW was calculated for each limb by weighing all the fruits on each limb and dividing by the number of fruits on that limb. Thus, there were three observations per tree. To calculate the true mean FW, the remaining fruits on each tree were harvested, counted, and weighed. The total weight for a tree was divided by the number of fruit on that tree. Fruits harvested for the 20-fruit sample and the limb samples were included in the calculation of the true mean.

Descriptive statistics for each method are presented in Table 2. The 20 -fruit sample represented an average of $5.8 \%$ of the fruit on the tree, whereas limbs contained $\approx 6.9 \%$ of the crop. Mean FW was 153,145 , and $150 \mathrm{~g}$ for the 20 -fruit sample, the limb sample, and the tree, respectively. The standard deviation and the range were greatest for the limb samples and least for the entire tree.

Although both sampling methods appear to provide fairly accurate estimates of $\mathrm{FW}$, when the estimated $\mathrm{FW}$ was regressed against the true mean for each tree, the coefficients of determination were only 0.77 and $0.86(P=0.001, \mathrm{n}=43)$ for the 20 -fruit sample and the limb sample, respectively. Based on other graphical and statistical techniques (unpublished data), a 20-fruit sample appears inadequate to accurately estimate average FW. A sample representing $\approx 25 \%$ of the crop would probably be adequate. The major problem with sampling fruit is that it is impossible to obtain a truly random sample. Although the limb sample is more expensive, it provides a larger sample size and seems to represent the tree better than sampling individual fruits. Sampling four or five limbs per tree likely would provide better estimates. Trees used in this experiment were relatively small and larger trees would likely require larger numbers of sub-samples.

Data analysis. The limb sampling method described above is a subsampling technique. Sub-sampling is common in pomological research and other examples include using more than one tree per plot, measuring the lengths of several shoots per tree, or measuring firmness on several fruits per tree. Some researchers perform statistical analyses on the mean of the three limbs for each tree; this provides one observation per tree. Such an approach works well when the experiment is balanced.

Table 2. Descriptive statistics for two methods of sampling a tree to estimate average fruit weight compared with the true mean obtained by harvesting all fruit on a tree.

\begin{tabular}{|c|c|c|c|}
\hline Sample unit & Mean & SD & Range \\
\hline \multicolumn{4}{|c|}{ Number of fruits sampled per tree } \\
\hline 20-fruit & 20 & 0 & $20-20$ \\
\hline Limbs & 29 & 16 & 4-99 \\
\hline Tree & 435 & 187 & $123-928$ \\
\hline \multicolumn{4}{|c|}{ Percentage of total number of fruits/tree } \\
\hline 20-fruit & 5.8 & 3 & $2-17$ \\
\hline Limbs & 6.9 & 3.1 & $4-23$ \\
\hline Tree & 100 & 0 & $100-100$ \\
\hline \multicolumn{4}{|c|}{ Fruit $w t(g)$} \\
\hline 20-fruit & 153 & 26.2 & $97-191$ \\
\hline Limbs & 145 & 29.0 & $79-223$ \\
\hline Tree & 150 & 24.1 & $101-191$ \\
\hline
\end{tabular}


Table 3. Influence of a factorial combination of four levels of oxamyl and four levels of naphthaleneacetic acid (NAA) on average fruit weight (g), estimated by harvesting all fruit on three limbs per tree. ${ }^{2}$

\begin{tabular}{lcccc}
\hline \hline $\begin{array}{l}\text { Oxamyl Conc. } \\
\left(\mathrm{mg} \cdot \mathrm{L}^{-1}\right)\end{array}$ & \multicolumn{4}{c}{ NAA Conc. $\left(\mathrm{mg} \cdot \mathrm{L}^{-1}\right)$} \\
\cline { 2 - 5 } & 0 & 2 & 4 & 6 \\
\hline 0 & 112 & 122 & 121 & 106 \\
250 & 140 & 143 & 146 & 133 \\
500 & 160 & 155 & 148 & 168 \\
750 & 162 & 147 & 183 & 171 \\
\hline
\end{tabular}

${ }^{2}$ Least squares means were calculated from 3 limbs from each of 3 trees per treatment.

For unbalanced experiments, including data for each limb is preferable and provides three observations per tree. Unfortunately, this experiment is unbalanced because the number of trees per treatment and the number of limbs per tree are not the same for every treatment. Often, this type of experiment is analyzed with an analysis of variance (ANOVA), using the Type III sums of squares, and means are separated with a multiple comparison such as Duncan's multiple range test or LSD, using the mean square error from the Type III sums of squares. Most statisticians would suggest comparing the least squares means, sometimes called "adjusted means", with a modification of the LSD, such as the Probability of the Difference (PDIFF), calculated with the Type III sums of squares. Least squares means are estimators of the marginal means that would be expected if the experiment had been balanced. A major problem in the analysis of unbalanced data is the contamination of differences betweenfactor means by effects of other factors. The solution to this problem is to adjust the means to remove the contaminating effects.

This type of experiment, with quantitative factors such as several concentrations of a growth regulator, is often analyzed with a one-way ANOVA and treatment means are compared with a multiple comparison. Such an approach does not provide as much information as a twoway ANOVA because there is no way to evaluate interaction. Using orthogonal polynomials also gives an indication of the nature of how a response variable is related to changing concentrations.

To demonstrate how different approaches to analyzing data may influence the conclusions, data from this experiment were analyzed three different ways. Examples of each of these approaches exist in the literature.

1) The general linear models (GLM) procedure of SAS (Littell, et al., 1991) was used to perform a two-way ANOVA, using means of the three limbs per tree (one observation per tree, $n=48$ ), to test the main effects and the interaction. A set of orthogonal polynomials was constructed to partition the degrees of freedom and sums of squares into components due to linear and quadratic regression.

2) The GLM procedure was used to perform a two-way ANOVA, using values of the three limbs per tree (three observation per tree, $\mathrm{n}=$ 132), to test the main effects and the interaction. A set of orthogonal polynomials was constructed to partition the degrees of freedom and sums of squares into components due to linear and quadratic regression.

3) The mixed procedure (Littell, et al., 1996) was used to perform a two-way ANOVA, using values of the three limbs per tree (three observation per tree, $n=132$ ), to test the main effects and the interaction. A set of orthogonal polynomials was constructed to partition the degrees of freedom and sums of squares into components due to linear and quadratic regression.

Least squares means are presented in Table 3. Fruit weight tended to increase with increasing concentration of oxamyl, but fruit weight was not consistently influenced by NAA.
Table 4. $P$-values from three different analyses of variance (ANOVA) for a factorial experiment with four levels of oxamyl and four levels of NAA. The design was completely randomized with three trees per treatment combination and three limbs per tree were sampled to estimate average fruit weight. The experiment was unbalanced because data for one tree and data for two limbs were not included in the analysis. ANOVAs and orthogonal polynomials were performed with SAS's GLM or Mixed Procedures. The analyses were performed on means of the three limbs per tree (no sampling) or using data from individual limbs (sampling).

\begin{tabular}{|c|c|c|c|}
\hline \multirow[b]{2}{*}{ Source of variation } & \multicolumn{3}{|c|}{ Procedure and sampling } \\
\hline & $\begin{array}{c}\text { GLM } \\
\text { no sampling }\end{array}$ & $\begin{array}{c}\text { GLM } \\
\text { sampling }\end{array}$ & $\begin{array}{c}\text { Mixed } \\
\text { sampling }\end{array}$ \\
\hline Oxamyl & 0.0001 & 0.0001 & 0.0001 \\
\hline Linear & 0.0001 & 0.0001 & 0.0001 \\
\hline Quadratic & 0.0286 & 0.1210 & 0.0231 \\
\hline NAA & 0.4974 & 0.7461 & 0.4424 \\
\hline Linear & 0.5174 & 0.6444 & 0.4907 \\
\hline Quadratic & 0.6546 & 0.7497 & 0.6335 \\
\hline Oxamyl $\times$ NAA & 0.0611 & 0.4652 & 0.0466 \\
\hline Oxamyl $_{\text {Linear }} \times \mathrm{NAA}_{\text {Linear }}$ & 0.1650 & 0.3234 & 0.1430 \\
\hline Oxamyl $_{\text {Linear }} \times$ NAA $_{\text {Quadratic }}$ & 0.4749 & 0.6105 & 0.4471 \\
\hline Oxamyl $_{\text {Quadratic }} \times \mathrm{NAA}_{\text {Linear }}$ & 0.0837 & 0.2193 & 0.0697 \\
\hline Oxamyl $_{\text {Quadratic }} \times \mathrm{NAA}_{\text {Quadratic }}$ & 0.3226 & 0.4811 & 0.2940 \\
\hline
\end{tabular}

Interpretations of the results of this experiment vary depending on which analysis is used (Table 4). Using the GLM procedure with no sampling indicates that FW increases linearly with increasing oxamyl and FW is not influenced by NAA and there is no oxamyl $\times$ NAA interaction. The analysis performed with the GLM procedure with sampling indicates that FW increases curvilinearly with increasing oxamyl concentration. NAA did not influence FW, but the interaction was significant at the $6 \%$ level and may deserve further investigation. The analysis performed with the Mixed procedure indicates that FW increases curvilinearly with increasing oxamyl concentration. NAA did not influence FW, but there is an important interaction and the oxamyl $_{\text {Quadratic }} \times \mathrm{NAA}_{\text {linear }}(P=0.07)$ interaction should be investigated further.

Until 1992, the second method was the most appropriate analysis that researchers could easily perform. However, the GLM procedure of SAS was developed to analyze "fixed effects" models. In this experiment we have a "mixed effects" model because the treatments are fixed but the limbs nested in trees (sub-samples) are random effects. When an experiment has mixed effects (such as blocks in a randomized complete-block design or a split-plot design) and the experiment is entirely balanced, with no missing observations, the means and the Ftests produced by the GLM procedure are valid. However, when there are missing observations in a mixed model, the least squares means, the standard errors of the least squares means, and the degrees of freedom may not be calculated correctly. Therefore, the F tests and any multiple comparisons may not be valid.

As we work to understand the biological and environmental factors influencing thinning results, we might also consider developing some protocols for designing thinning experiments, data collection, and data analysis and summarization. We should not expect to obtain similar results until we conduct our experiments in a similar manner.

\section{Literature Cited}

Littell, R.C., R.J. Freund, and P.C. Spector. 1991. SAS system for linear models. $3^{\text {rd }}$ ed. SAS Inst. Inc., Cary, NC.

Littell, R.C., G.A. Milliken, W.W. Stroup, and R.D. Wolfinger. 1996. SAS system for mixed models. SAS Inst. Inc., Cary N.C. 